\title{
ASYMPTOTIC DISTRIBUTION OF SUMS OF RADEMACHER FUNCTIONS
}

\author{
JEROME C. SMITH
}

1. Introduction. The central limit theorem of the calculus of probability applied to the special case of the Rademacher functions $r_{k}(t)=\operatorname{sign}\left(\sin 2^{k} \pi t\right)$ can be stated as follows :

$$
\lim _{n \rightarrow \infty}\left|\underset{t}{E}\left\{\frac{1}{(2 n)^{1 / 2}}\left|\sum_{k=1}^{n} r_{k}(t)\right|<a\right\}\right|=\frac{1}{\pi^{1 / 2}} \int_{-a}^{a} e^{-u^{2}} d u .
$$

This form of the theorem suggests two generalizations, the first of which consists in replacing the constant $a$ by a function $f(t)$. The second generalization is obtained by replacing the constant upper limit of summation $n$ by a sequence of integral-valued functions $N_{n}(t)$, so that the number of Rademacher functions in the sum varies with $t$. These are proved by combining the standard techniques of the calculus of probability with the methods of orthogonal series. Both proofs make use of the continuity theorem of Fourier-Stieltjes transforms:

$$
\begin{aligned}
& \text { If } f(t)=\lim _{n \rightarrow \infty} f_{n}(t), \text { and if } \\
& \qquad \lim _{n \rightarrow \infty} \int_{0}^{1} \exp \left[i x f_{n}(t)\right] d t=e^{-x^{2} / 4}
\end{aligned}
$$

uniformly in $x$, then

$$
\lim _{n \rightarrow \infty}\left|\underset{t}{E}\left\{\left|f_{n}(t)\right|<a\right\}\right|=\frac{1}{\pi^{1 / 2}} \int_{-a}^{a} e^{-u^{2}} d u .
$$

Essential use is made of the Walsh-Kaczmarz system ${ }^{1}$ of orthonormal functions, which have two useful properties: (1) each of them is equal to a finite product of Rademacher functions, and (2) any Lebesgue-square integrable function can be expanded in a series of these functions, which series will converge in the mean with index two.

2. First generalization. The first theorem expresses in asymptotic form the measure of the point set over which the sum of the Rademacher functions is bounded by a given function.

Received by the editors June 25,1945 . The material of this paper forms part of a thesis, prepared under the guidance of Professor Mark Kac, which was presented to the Graduate School of Cornell University for the degree of Doctor of Philosophy.

${ }^{1}$ Cf. S. Kaczmarz and H. Steinhaus, Le systeme orthogonal de M. Rademacher, Studia Mathematica vol. 2 (1930) and J. L. Walsh, A closed set of normal orthogonal functions, Amer. J. Math. vol. 45 (1923) pp. 5-24. 
THEOREM. If $f(t)$ is a non-negative, measurable function in the interval $(0,1)$, then

$$
\lim _{n \rightarrow \infty}\left|\underset{t}{E}\left\{\frac{1}{(2 n)^{1 / 2}}\left|\sum_{k=1}^{n} r_{k}(t)\right|<f(t)\right\}\right|=\frac{1}{\pi^{1 / 2}} \int_{0}^{1} \int_{-f(t)}^{f(t)} e^{-u^{2}} d u d t .
$$

PRoof. Let $F_{n}(t)$ be $(2 n)^{-1 / 2} \sum_{k=1}^{n} r_{k}(t)$ and let $S_{r}(t)$ be the partial sum of the expansion of a Lebesgue-square integrable function $h(t)$ in a series of Walsh-Kaczmarz functions. It follows from the first property of these functions that

$$
\lim _{n \rightarrow \infty} \int_{0}^{1} S_{r}(t) \exp \left[i x F_{n}(t)\right] d t=e^{-x^{2} / 4} \int_{0}^{1} h(t) d t .
$$

In view of the second property of the Walsh-Kaczmarz functions, this implies that

$$
\lim _{n \rightarrow \infty} \int_{0}^{1} h(t) \exp \left[i x F_{n}(t)\right] d t=e^{-x^{2} / 4} \int_{0}^{1} h(t) d t .
$$

If $h(t)$ be the characteristic function of an interval $E$, then

$$
\lim _{n \rightarrow \infty} \int_{E} \exp \left[i x F_{n}(t)\right] d t=|E| \cdot e^{-x^{2} / 4} .
$$

By virtue of the continuity theorem of Fourier-Stieltjes transforms, it can be demonstrated that

$$
\lim _{n \rightarrow \infty}\left|\underset{t}{E}\left\{t \in E ;\left|F_{n}(t)\right|<a\right\}\right|=\frac{|E|}{\pi^{1 / 2}} \int_{-a}^{a} e^{-u^{2}} d u .
$$

Hence, if $g(t)$ be a non-negative step-function having the constant value $a_{k}$ over the interval $E_{k}$, then

$$
\lim _{n \rightarrow \infty}\left|\underset{t}{E}\left\{\left|F_{n}(t)\right|<g(t)\right\}\right|=\lim _{n \rightarrow \infty} \sum_{k=1}^{\infty}\left|\underset{t}{E}\left\{t \in E_{k} ;\left|F_{n}(t)\right|<a_{k}\right\}\right| .
$$

Inspection shows that the limit on the right is equal to

$$
\frac{1}{\pi^{1 / 2}} \int_{0}^{1} \int_{-o(t)}^{o(t)} e^{-u^{2}} d u d t
$$

The theorem now follows by considering $f(t)$ to be the limit of properly chosen step-functions.

3. Second generalization. In order to obtain the generalization of the central limit theorem in which the upper limit of summation of 
the Rademacher functions is a given function, we must properly qualify this function.

THEOREM. Let $N_{n}(t)=n N(t)+Q_{n}(t)$, where $N_{n}(t)$ and $N(t)$ assume as values only zero and the positive integers and where $N(t)$ is Lebesgue integrable, with $p=\int_{0}^{1} N(t) d t$. If $\lim _{n \rightarrow \infty} n^{-1 / 2}\left|Q_{n}(t)\right|=0$, then for any non-negative $a$,

$\lim _{n \rightarrow \infty}\left|\underset{t}{E}\left\{\frac{1}{(2 n p)^{1 / 2}}\left|\sum_{k=1}^{N_{n}(t)} r_{k}(t)\right|<a\right\}\right|=\left(\frac{p}{\pi}\right)^{1 / 2} \sum_{M=0}^{\infty} \frac{\left|E_{M}\right|}{M^{1 / 2}} \int_{-a}^{a} e^{-p x^{2} / M} d x$

where

$$
E_{M}=\underset{t}{E}\{N(t)=M\}
$$

Proof. Since the conditions of the theorem imply that

$$
\sum_{k=1}^{N_{n}(t)} r_{k}(t)=\sum_{k=1}^{n N(t)} r_{k}(t)+S_{n}(t)
$$

where $S_{n}(t)$ is such that $\left|S_{n}(t)\right| \leqq\left|Q_{n}(t)\right|$, it follows that

$$
\begin{aligned}
\lim _{n \rightarrow \infty} \int_{E_{M}} \exp \left[\frac{i x}{(2 n p)^{1 / 2}} \sum_{k=1}^{N_{n}(t)} r_{k}(t)\right] d t & \\
& =\lim _{n \rightarrow \infty} \int_{E_{M}} \exp \left[\frac{i x}{(2 n p)^{1 / 2}} \sum_{k=1}^{n N(t)} r_{k}(t)\right] d t .
\end{aligned}
$$

Since $N(t)$ is constant over $E_{M}$, the second limit equals $\left|E_{M}\right| \cdot e^{-M x^{2} / 4 p}$. By virtue of the continuity theorem of Fourier-Stieltjes transforms, it follows that

$$
\begin{aligned}
\lim _{n \rightarrow \infty}\left|\underset{t}{E}\left\{t \in E_{M} ; \frac{1}{(2 n p)^{1 / 2}}\left|\sum_{k=1}^{N_{n}(t)} r_{k}(t)\right|<a\right\}\right| \\
=\left|E_{M}\right| \cdot\left(\frac{p}{M \pi}\right)^{1 / 2} \int_{-a}^{a} e^{-p x^{2} / M} d x,
\end{aligned}
$$

and hence the theorem is proved.

The conclusion of the theorem obviously holds under the stronger condition that there exists an $N(t)$ and an $A$ such that $\left|N_{n}(t)-n N(t)\right|$ $<A$ for all $n$.

4. Sums of cosines with big gaps. By a similar method, using a Fourier series instead of a series of Walsh-Kaczmarz functions, it can be proved ${ }^{2}$ that if $f(t)$ is a positive, measurable function in the inter-

2 Professor M. Kac has proved the central limit theorem for cosines with big gaps in Note on power series with big gaps, Amer. J. Math. vol. 61 (1939) pp. 473-476. 
val $(0,1)$ and if $\left\{n_{k}\right\}$ is a sequence of positive integers satisfying the gap condition : $\lim _{k \rightarrow \infty} n_{k} / n_{k-1}=\infty$, then

$$
\lim _{m \rightarrow \infty}\left|\underset{t}{E}\left\{\frac{1}{m^{1 / 2}}\left|\sum_{k=1}^{m} \cos 2 \pi n_{k} t\right|<f(t)\right\}\right|=\frac{1}{\pi^{1 / 2}} \int_{0}^{1} \int_{-f(t)}^{f(t)} e^{-u^{2}} d u d t .
$$

CoRnell University 